\title{
A large microRNA cluster on chromosome 19 is a transcriptional hallmark of WHO type $A$ and $A B$ thymomas
}

Milan Radovich*,1, Jeffrey P Solzak ${ }^{1}$, Bradley A Hancock ${ }^{1}$, Madison L Conces ${ }^{2}$, Rutuja Atale ${ }^{1}$, Ryan F Porter ${ }^{2}$, Jin Zhu ${ }^{3}$, Jarret Glasscock ${ }^{3}$, Kenneth A Kesler ${ }^{1}$, Sunil S Badve ${ }^{4}$, Bryan P Schneider ${ }^{2}$ and Patrick J Loehrer ${ }^{2}$

${ }^{1}$ Department of Surgery, Indiana University School of Medicine, 980 W Walnut Street, Room C312, Indianapolis, IN 46202, USA; ${ }^{2}$ Department of Medicine, Division of Hematology/Oncology, Indiana University School of Medicine, 980 W Walnut Street, Room C312, Indianapolis, IN 46202, USA; ${ }^{3}$ Cofactor Genomics, LLC, 4044 Clayton Avenue, St Louis, MO 63110, USA and ${ }^{4}$ Department of Pathology and Laboratory Medicine, Indiana University Melvin and Bren Simon Cancer Center, Indiana University School of Medicine, 980 W Walnut Street, Room C312, Indianapolis, IN 46202, USA

Background: Thymomas are one of the most rarely diagnosed malignancies. To better understand its biology and to identify therapeutic targets, we performed next-generation RNA sequencing.

Methods: The RNA was sequenced from 13 thymic malignancies and 3 normal thymus glands. Validation of microRNA expression was performed on a separate set of 35 thymic malignancies. For cell-based studies, a thymoma cell line was used.

Results: Hierarchical clustering revealed 100\% concordance between gene expression clusters and WHO subtype. A substantial differentiator was a large microRNA cluster on chr19q13.42 that was significantly overexpressed in all $A$ and $A B$ tumours and whose expression was virtually absent in the other thymomas and normal tissues. Overexpression of this microRNA cluster activates the PI3K/AKT/mTOR pathway. Treatment of a thymoma AB cell line with a panel of PI3K/AKT/mTOR inhibitors resulted in marked reduction of cell viability.

Conclusions: A large microRNA cluster on chr19q13.42 is a transcriptional hallmark of type A and AB thymomas. Furthermore, this cluster activates the PI3K pathway, suggesting the possible exploration of PI3K inhibitors in patients with these subtypes of tumour. This work has led to the initiation of a phase II clinical trial of PI3K inhibition in relapsed or refractory thymomas (http://clinicaltrials.gov/ct2/show/NCT02220855).

Thymoma is a rare disease with $\sim 400$ cases diagnosed in the United States per year (Engels, 2010). Though considered by some to be benign, all subtypes of thymoma have been reported to be invasive and capable of metastasis (Jain et al, 2010; Vladislav et al, 2012). Although untreated thymomas are uniquely sensitive to chemotherapy, standard chemotherapy alone is not curative. Surgical management is highly beneficial for those with resectable disease, but 10-29\% will recur after initial therapy (Hamaji et al, 2014). Thymomas are histologically characterised into several World Health Organisation (WHO) subtypes based on epithelial cell morphology and lymphocytic infiltration. These include the A and $A B$ subtypes defined by spindle/oval epithelial cell morphology ( $\mathrm{AB}$ includes dense lymphocytic foci); B1 that has a round epithelioid shape with a predominance of lymphocytes; B2 that has a polygonal shape with lymphocytic infiltration; B3 that is defined by cells with a round or polygonal epithelioid shape and a small lymphocytic component; and $\mathrm{C}$ that is defined as thymic carcinoma with histological features common of epithelial cancers (Dadmanesh et al, 2001; Travis et al, 2004). Though generally accepted, this classification has its detractors based on complexity,

*Correspondence: Dr M Radovich; E-mail: mradovic@iupui.edu

Received 8 March 2015; revised 3 November 2015; accepted 10 November 2015; published online 14 January 2016

(c) 2016 Cancer Research UK. All rights reserved 0007-0920/16 
interobserver inconsistency, sampling errors (in which multiple subtypes are seen in larger specimens), and lack of prognostic consistency (Moran and Suster, 2008; Wick, 2008, 2010; Zucali et al, 2013). Furthermore, patients with advanced thymoma are typically treated with systemic therapy, independent of histologic classification. Published molecular data to date have been unable to identify recurrent DNA mutations or somatic copy number alterations that have correlated with histologic subtype or clinicopathologic features, but have revealed molecular distinctions between thymoma and thymic carcinoma, suggesting that thymomas are a separate entity from thymic carcinomas (Girard et al, 2009). Little is known about the biology of these rare thymoma tumours, in particular, the extent of dysregulation present in the transcriptional landscape. To examine this landscape, next-generation RNA sequencing (RNA-seq) was performed to profile the entire transcriptome of these tumours. Because of the comprehensive nature of this technology, measurement of the gene expression of all protein coding as well as noncoding RNAs, in particular precursor microRNAs, was completed. These data were then analysed to determine whether novel biological hallmarks could lend clues to biomarkers and potential treatments for this disease.

\section{MATERIALS AND METHODS}

Samples and RNA-sequencing. Frozen tissue from 13 thymic malignancies and 3 normal tissues used for RNA-seq were obtained from the Indiana University Simon Cancer Center Tissue Bank. Samples were WHO subtyped by an experienced pathologist (SB) in a blinded manner. Because thymic malignancies can have a pervasive lymphocytic presence, we chose tumour tissues that were lymphocyte poor and contained high tumour cellularity in order to enrich for tumour-specific RNA. The WHO subtypes represented include: (4) type A, (2) AB, (1) B2, (5) B3, (1) C, and (3) normal tissues (sample details in Supplementary Table 1). RNA was extracted using the Ambion Ribopure extraction kit (Life Technologies, South San Francisco, CA, USA) followed by ribosomal depletion using the Ribominus Eukaryote Kit (Life Technologies). The RNA-seq libraries were prepared using the Total RNA-Seq Kit (Life Technologies). Emulsion PCR and bead preparation was conducted according to the manufacturer's instructions. Each library was barcoded to allow multiple samples to be sequenced simultaneously. The $16 \mathrm{cDNA}$ libraries were sequenced on a Life Technologies SOLiD 3-plus and SOLiD 4 sequencers using two $50 \mathrm{bp}$ fragment runs and one $75 \times 35 \mathrm{bp}$ paired-end run (Alignment information in Supplementary Table 2). For gene expression, mapping of reads to the genome (hg19) was performed using the Life Technologies LifeScope 2.5.1 pipeline and output BAM (Binary Alignment/ Mapping) files imported into Partek Genomics Suite for analysis. BAM files to be submitted to the NCBI Gene Expression Omnibus (GEO) for public availability. In Partek, mapped reads were crossreferenced against known genes from the RefSeq database followed by statistical comparison of RPKM (reads per kilobase of exon per million mapped reads) values for each gene. In addition, mapped reads were also cross-referenced against a database of precursor microRNAs downloaded from miRBase version 20 (ftp://mirbase.org/pub/mirbase/CURRENT/genomes/hsa.gff3) (Kozomara and Griffiths-Jones, 2014). Unsupervised hierarchical clustering analyses using Spearman's correlation with average linkage were performed using Partek and network analyses were performed using Ingenuity Pathway Analysis (Ingenuity Systems, Redwood City, CA, USA).

Validation sample set and qPCR. For miRNA validation, a larger independent set of 35 thymic malignancies was used. RNA was extracted from these tissues using the All Prep RNA/DNA/Protein kit with an additional miRNA enrichment step as per the manufacturer's protocol (Qiagen, Valencia, CA, USA). Samples were reverse transcribed using the microRNA Reverse Transcription Kit (Life Technologies). Validation of miRNA expression was performed by qPCR using a custom miRNA TaqMan low-density array with the Life Technologies 7900HT Real-Time PCR System. RNU24, RNU44, and RNU48 were used as housekeeper genes. The qPCR data were analysed using the SDS2.3 and DataAssist v3.01 software from Life Technologies. All studies on all samples were approved by the IU Institutional Review Board.

Protein analyses. Protein measurements in thymoma tissues for PTEN and phospho-AKT (p-Ser 473) were performed using enzyme-linked immunosorbent assays (ELISA) (R\&D Systems, Minneapolis, MN, USA). Statistical analyses were performed using Prism 6.0 software (GraphPad Software, La Jolla, CA, USA) with Student's two-sided two-sample $t$-test. For cell-based experiments, we used a previously described thymoma $\mathrm{AB}$ cell line (IU-TAB1) developed at Indiana University (Indianapolis, IN, USA; Gokmen-Polar et al, 2012). Protein analysis of IU-TAB1 cells before and after transfection with human mir-517a and mir-519d (Ambion, Austin, TX, USA) was executed using western blot. Cells $(n=300000)$ were seeded in 6-well plates and transfected with the microRNAs individually and in combination for $60 \mathrm{~h}$. Protein was isolated and quantified using RIPA buffer and BCA assay (Pierce, Rockford, IL, USA). The protein lysate $(20 \mu \mathrm{g})$ was run on a precast $10 \%$ Bis-Tris gel (Life Technologies) and wet transferred to a PVDF membrane. After blocking, membranes were probed with PTEN, phospho-AKT (Ser 473), AKT (pan-AKT1/2/3), phospho-mTOR (Ser 2448), mTOR, phospho-p70 S6K (Ser 371), phospho-4E-BP1 (Thr 37/46), and $\beta$-actin (Cell Signaling Technology, Danvers, MA, USA) antibodies. Detection was performed using SuperSignal West Pico Chemiluminescent Substrate (Pierce) and imaging was performed on a Fujifilm LAS 4000 imager (Fujifilm, Valhalla, NY, USA).

Drug sensitivity experiments. IU-TAB1 cells $(n=10000)$ were seeded in 96-well plates and dosed with increasing concentrations of $\mathrm{PI} 3 \mathrm{~K}$ and PI3K/mTOR inhibitors. Measurement of cell viability was performed using the Promega CellFlour assay (Madison, WI, USA) using a Bio-Tek (Winooski, VT, USA) Synergy 4 multi-function plate reader. All experiments were performed with triplicate technical replicates, and each experiment performed three times. The $\mathrm{IC}_{50}$ values were calculated using Prism 6.0 software.

\section{RESULTS}

RNA-seq of protein coding genes confirms WHO histological subtypes. In our first analysis, we performed unsupervised hierarchical clustering using all expressed protein coding genes in these samples. As seen in Figure 1A, the dendrogram demonstrates a separation of samples based on gene expression in 100\% concordance with the WHO histological subtype. In particular, the $\mathrm{A}$ and $\mathrm{AB}$ samples separate from the B3 samples. The lymphocyte-rich B2/B3 sample separates from the $\mathrm{A}, \mathrm{AB}$, and $\mathrm{B} 3$ samples and is concordant with the lymphocyte-rich normal thymus tissues. The lone $\mathrm{C}$ sample (which is histologically defined as a thymic carcinoma, not thymoma) also separates into its own cluster. These data demonstrate that the WHO histological subtypes mirror the underlying transcriptional differences. We then compared the gene expression values of the $\mathrm{A}$ and $\mathrm{AB}$ samples $v s$ the other samples and observed that many of the most significant differentially expressed genes were pre-miRNAs all coming from a single cluster on chromosome 19q13.42 (Supplementary Table 3).

Precursor miRNA analysis defines subtypes and identifies overexpressed cluster. Because the RefSeq database is not a comprehensive annotation of microRNAs, we then analysed the data using a database of precursor microRNAs downloaded from the UCSC (University of California, Santa Cruz) genome browser. 

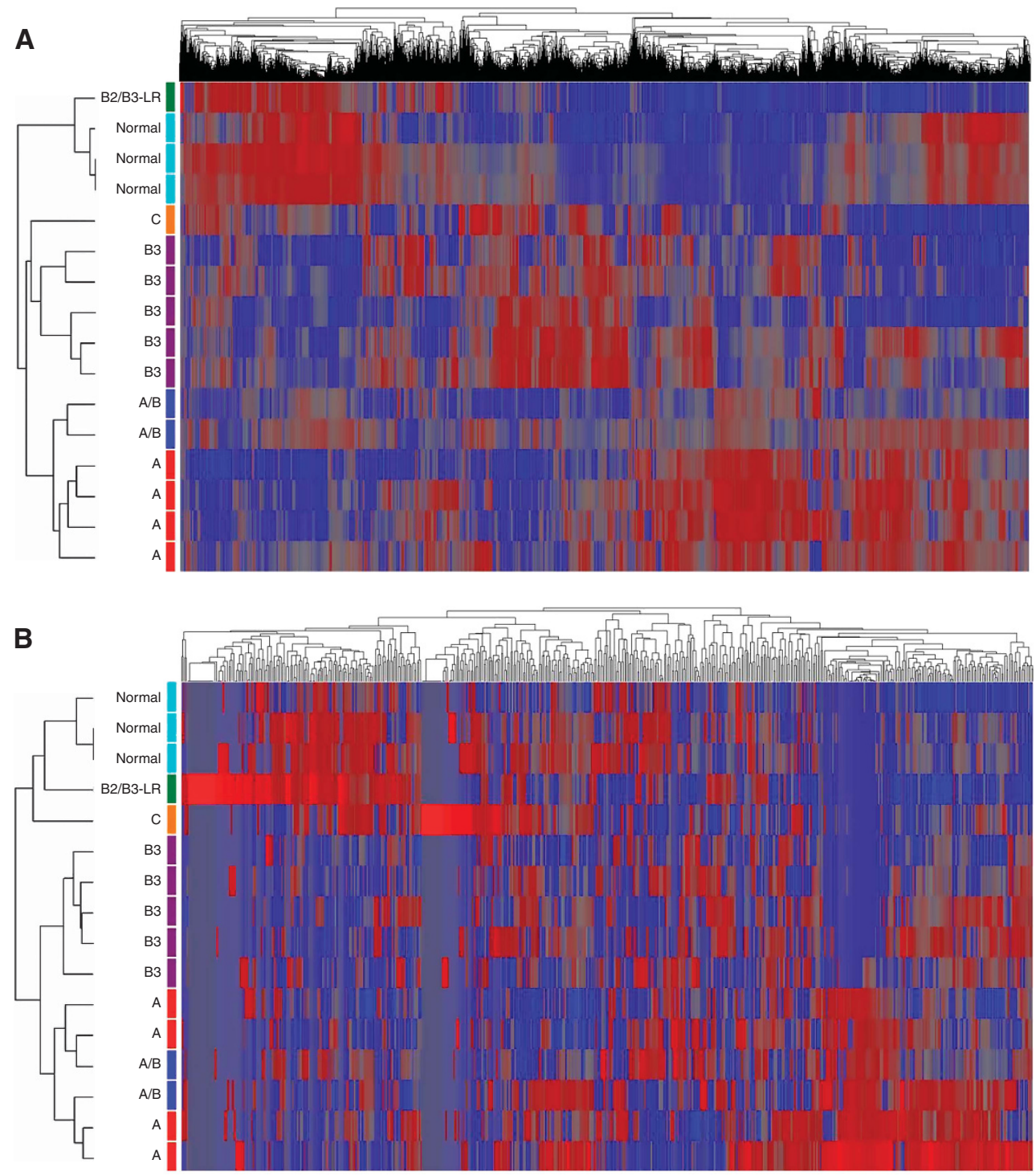

Figure 1. Hierarchical clustering of thymoma samples. (A) Unsupervised hierarchical clustering of all expressed RefSeq genes in the thymoma and normal samples by RNA-Seq. Clustering was performed using RPKM values of expressed genes. The dendrogram demonstrates separation of the samples based on gene expression that is completely concordant with the WHO histological subtype (LR=lymphocyte rich). Red indicates higher expression and blue indicates lower expression. (B) Unsupervised hierarchical clustering of all expressed precursor microRNAs (as defined by the UCSC genome browser) in the thymoma and normal samples. The dendogram demonstrates a mixing of the $A$ and $A B$ thymomas with separation of the other subtypes.

Because standard RNA-seq chemistry only measures longer length RNAs, we focussed the analysis on the longer precursor form with validation of the mature form using $\mathrm{qPCR}$. As seen in Figure 1B, unsupervised hierarchical clustering of expressed pre-miRNAs separated the samples based on WHO histological subtypes, similar to what was seen with protein coding genes, but with the $\mathrm{A}$ and $\mathrm{AB}$ samples mixed in a single dendrogram cluster. Differential expression analysis again revealed many of the most significant genes originating from a single microRNA cluster on chromosome 19q13.42 (C19MC) (Supplementary Table 4). A deeper analysis of this cluster revealed high overexpression of these microRNA in A and $\mathrm{AB}$ thymomas and virtually absent expression in the other samples (Table 1). To validate this observation, we performed qPCR for the miRNA cluster in a separate set of 35 thymic malignancies. The qPCR assays were designed for the mature version of the miRNAs. As seen in Figure 2, qPCR demonstrated a strong separation of our samples into two groups based on miRNA expression. All $\mathrm{A}$ and $\mathrm{AB}$ samples were classified into the group positive for $\mathrm{C} 19 \mathrm{MC}$ cluster, whereas the B1, B2, and B3 samples were present in the C19MC-negative group. Of note, only one discordant sample was present, a B1 thymoma, and it was classified into the C19MC cluster-positive group.

C19MC modulation of the PI3K/AKT pathway. Previous work in hepatocellular carcinoma has demonstrated that the C19MC cluster causes activation of the PI3K/AKT pathway by inhibition of the PI3K antagonist PTEN, and also of the cell cycle inhibitor p21 (Fornari et al, 2012). To investigate this, we performed a pathway analysis of the PI3K/AKT pathway and overlaid genes that were differentially expressed between $\mathrm{A}$ and $\mathrm{AB}$ vs $\mathrm{B} 3$ thymomas (false discovery rate $(\mathrm{FDR})<25 \%)$. As seen in Figure $3 \mathrm{~A}$, PI3K was significantly overexpressed in $\mathrm{A}$ and $\mathrm{AB}$ thymomas along with PREX2 (a PTEN antagonist; Fine et al, 2009), Ras, and MAGI. In addition, there was significant downregulation of the FOXO family of transcription factors, well known to be inactivated when PI3K/ AKT is activated (Brunet et al, 1999), along with BIM, p21cip1, and MAST2. To validate the pathway analysis, we performed protein measurements for phospho-AKT (Ser 473) and PTEN by ELISA on 
Table 1. Median RPKM expression of precursor microRNAs in the C19MC cluster grouped by WHO subtype

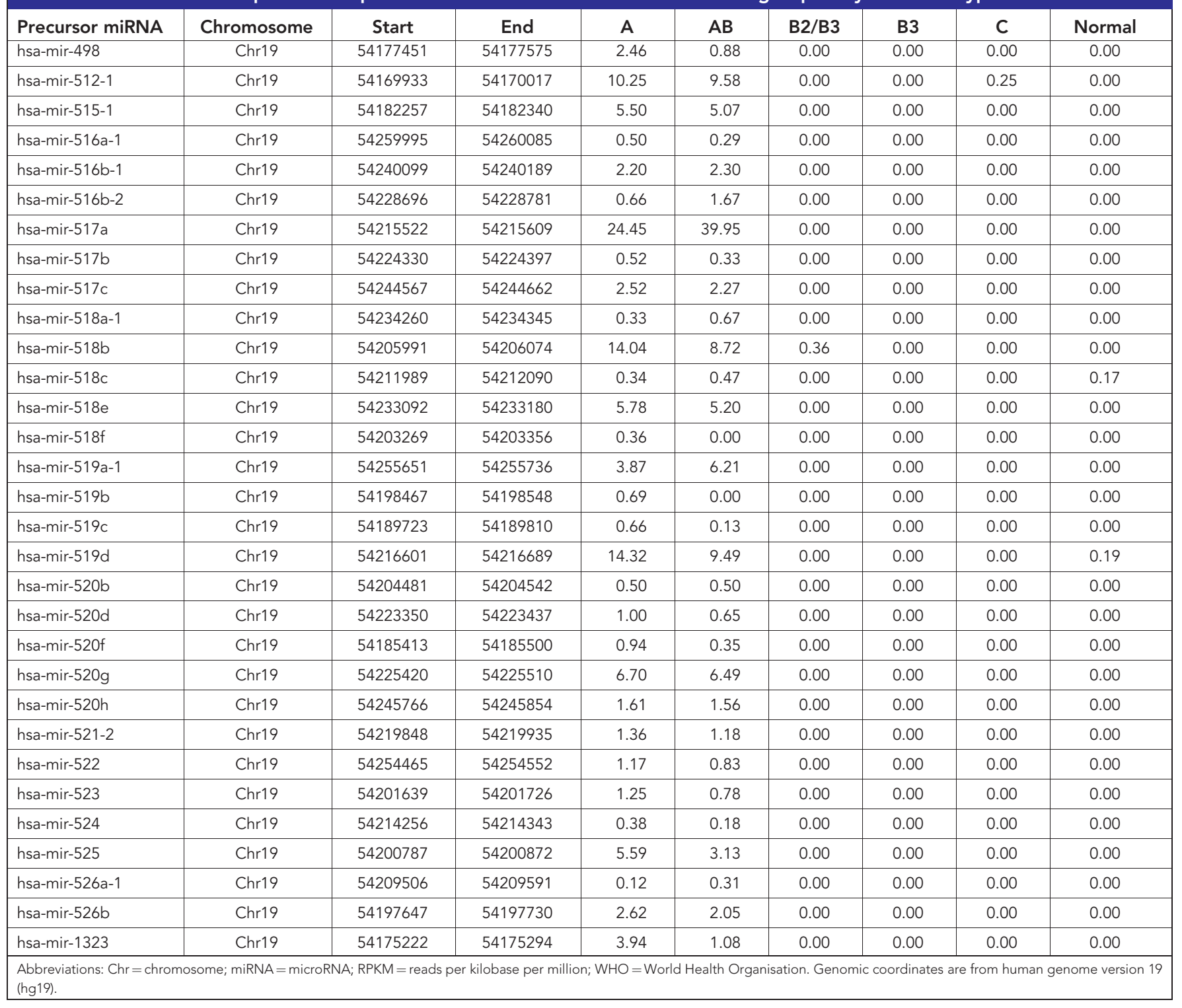

the same 35 thymomas used in the previous miRNA qPCR validation (Figure $3 \mathrm{~B}$ ). In congruence with the pathway analysis, we observed significantly higher activated phospho-AKT in the C19MC-positive thymomas as compared with the C19MCnegative tumours $(P<0.0001)$ as well as the expected downregulation of PTEN in the C19MC-positive thymomas as compared with the C19MC-negative tumours $(P<0.0001)$.

To provide additional support that the C19MC cluster induces activation of the PI3K pathway, we performed an in vitro experiment using the only known existing human Thymoma $\mathrm{AB}$ cell line, IU-TAB1. IU-TAB1 is a well-characterised cell line of $A B$ thymoma origin, though expression of the C19MC cluster is low. Two C19MC miRNAs (mir-519d and mir-517a) were transfected into the IU-TAB1 cell line. These two miRNAs are known or predicted regulators of PTEN, respectively (Fornari et al, 2012; Kozomara and Griffiths-Jones, 2014). Thymoma cells were transfected with the microRNAs individually as well as in combination. As seen in Figure 4, the transfection of the combination of mir-517a and mir-519d decreased protein expression of PTEN along with a corresponding increase in p-AKT and p-70 S6K. Upregulation of p-AKT, p-MTOR, p-70 S6K, and p-4EBP1 was also observed with mir-517a alone.
Sensitivity to PI3K pathway inhibitors. To demonstrate potential sensitivity of thymomas to PI3K pathway inhibitors, IU-TAB1 cells were dosed with increasing concentrations of 8 different PI3K or dual $\mathrm{PI} 3 \mathrm{~K} / \mathrm{mTOR}$ inhibitors that have been previously tested in phase 2 or phase 3 clinical trials. As seen in Figure 5, we observe significant sensitivity of this cell line to these inhibitors, with PF04691502 and BEZ235 demonstrating the best reduction in cell viability at an $\mathrm{IC}_{50}$ of 118 and $210 \mathrm{~nm}$, respectively. Both of these drugs are dual PI3K/mTOR inhibitors, and this may explain their increased potency compared with the other inhibitors tested.

\section{DISCUSSION}

Herein, we demonstrate that a large microRNA cluster on chromosome 19q13.42 (C19MC) is a genomic hallmark of type $\mathrm{A}$ and $\mathrm{AB}$ thymomas. This cluster is known to be the largest microRNA cluster in the human genome whose presence is restricted to primates (Zhang et al, 2008; Flor and Bullerdiek, 2012). This cluster is normally expressed only in embryonic development and is normally silent in adult tissues except the 


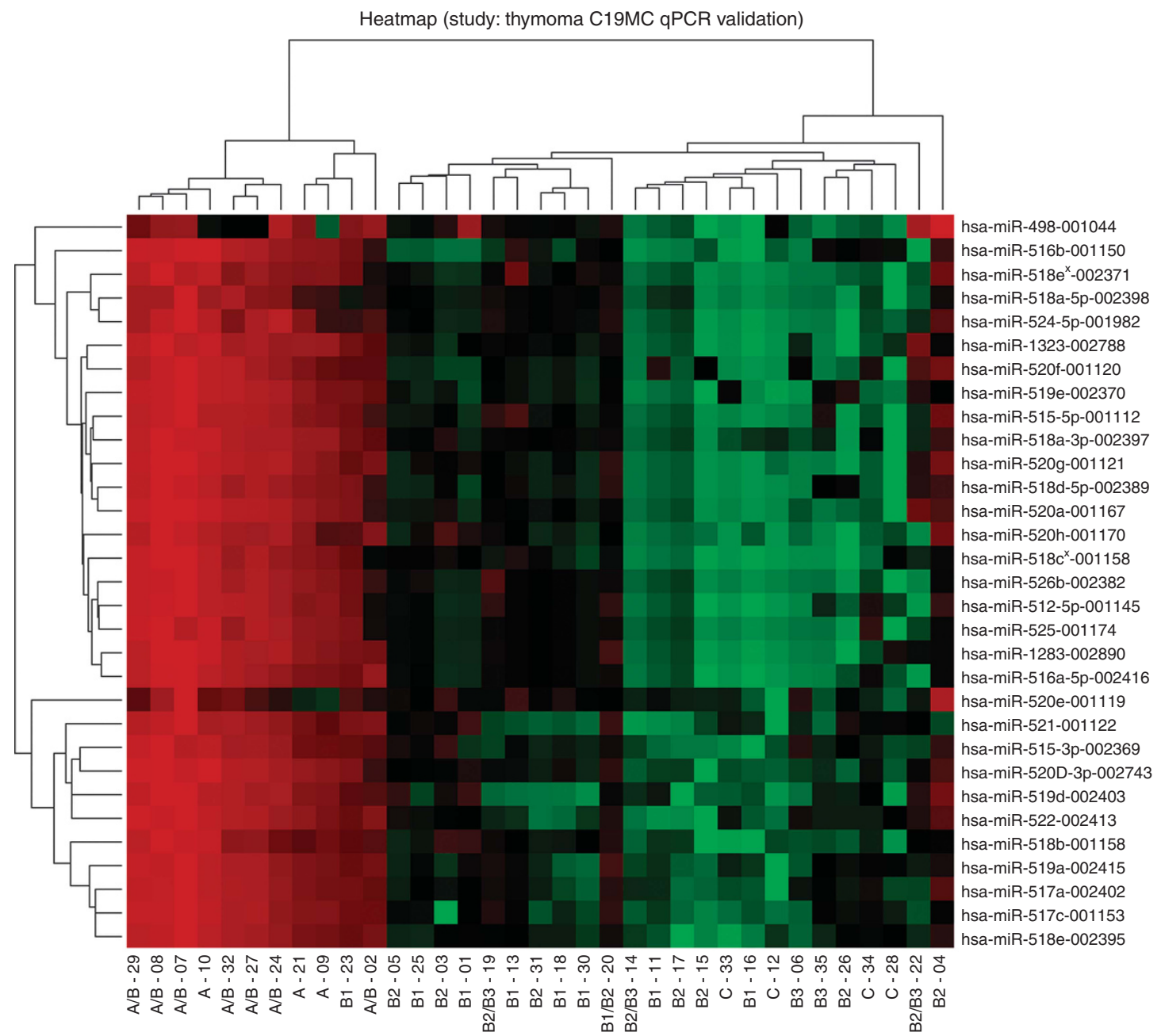

Figure 2. Validation of expression of the C19MC cluster by qPCR in an independent sample set of 35 thymomas. Expression data demonstrate a separation of the samples into two distinct groups. All A and AB samples were in the C19MC-positive group, whereas the B1, B2, and B3 samples were in the C19MC-negative group, except for one discordant B1 sample in the C19MC-positive group. Two-digit numbers after the WHO subtype below the map are the sample numbers (from Supplementary Table 1). Six-digit values on the right side of the heatmap after the microRNA gene symbol represent the miRNA qPCR assay IDs from Life Technologies.

placenta (Zhang et al, 2008; Bullerdiek and Flor, 2012). A search for other cancers revealed other tumour types that overexpress this cluster. Of interest, ETANTRs (embryonal tumours with abundant neuropil and true rosettes), a rare and highly aggressive primitive neuroendocrine tumour, overexpress this cluster in $93 \%$ of cases (Korshunov et al, 2010). In ETANTRs, overexpression of this cluster is mediated via somatic gene amplification (Korshunov et al, 2010). In a similarly classified set of tumours, ETMRs (embryonal tumours with multilayered rosettes), 12 out of 12 ETMRs demonstrated overexpression of C19MC driven by a fusion of the TTYH1 gene promoter (tweety family member 1) with C19MC, suggesting that a translocation along with amplification drives expression in these rare tumours (Kleinman et al, 2014). Overexpression of this cluster is similarly seen in thyroid adenomas (Rippe et al, 2010), parathyroid adenomas (Vaira et al, 2012), hepatic mesenchymal hamartomas (Kapur et al, 2014), hepatocellular carcinomas (Fornari et al, 2012), and in a subset of tamoxifen-resistant breast cancers (Kapur et al, 2014), mediated by a variety of mechanisms including gene amplification, chromosomal translocations, and hypomethylation. The mechanism of overexpression of this cluster in thymoma is currently unknown, but data from The Cancer Genome Atlas (TCGA) thymoma project will hopefully shed light onto the mechanism when the data become available. Of further interest to thymoma, it has been recently hypothesised that $\mathrm{C} 19 \mathrm{MC}$ exosomes are potent immunomodulators that may suppress immune function in the tumour microenvironment (Bullerdiek and Flor, 2012). Given the prevalence of autoimmune diseases in patients with thymoma (myasthenia gravis, red cell aplasia, Good's syndrome, and others; Shelly et al, 2011), a link between C19MC and autoimmunity is worth further exploration.

One of the key functions of this microRNA cluster is the overactivation of the PI3K/AKT pathway (Fornari et al, 2012). Although overactivation of this pathway is common in many cancers, the mean of activation varies, but in many cases is achieved via somatic mutations in PIK3CA or PTEN. This does not seem to be the case for thymoma where previous work has demonstrated a lack of mutations in these genes (Girard et al, 2009). To confirm overactivation, pathway analysis demonstrated differentially expressed genes consistent with an activated PI3K/ AKT pathway in our type $\mathrm{A}$ and $\mathrm{AB}$ thymomas. Further proteinlevel data in our validation cohort demonstrated elevated p-AKT and decreased PTEN in our C19MC-positive tumours. To translate these findings, we used an established thymoma $\mathrm{AB}$ cell line (Gokmen-Polar et al, 2012) and tested a variety of PI3K and dual $\mathrm{PI} 3 \mathrm{~K} / \mathrm{mTOR}$ inhibitors that have been tested in cancer clinical trials. Recently, PI3K and dual PI3K inhibitors have shown promise as potential clinical agents (Rodon et al, 2013). 

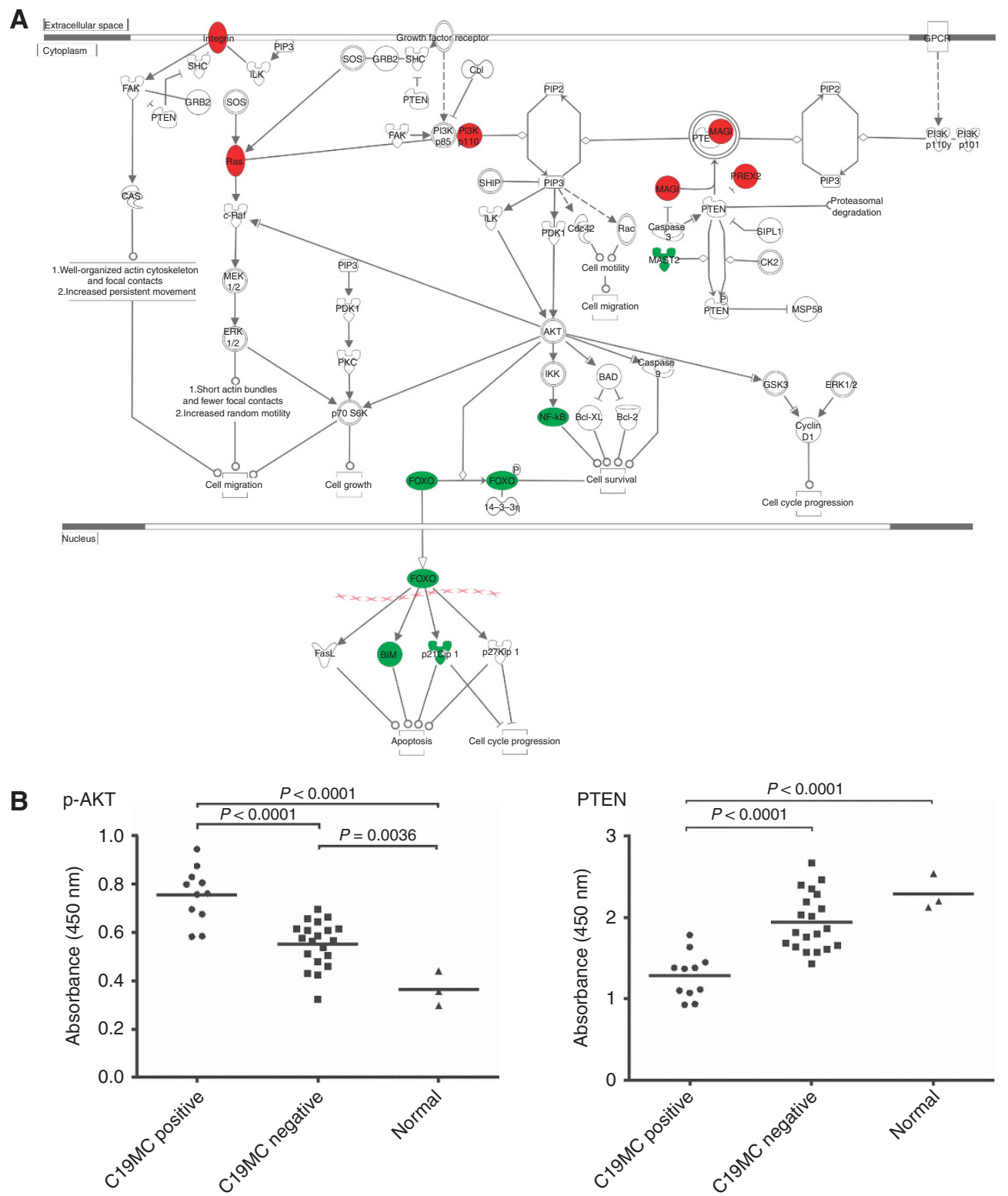

Figure 3. PI3K pathway analysis among thymoma subtypes. (A) Network analysis demonstrates overexpressed and underexpressed genes in A and $A B$ thymomas vs $B 3$ thymomas (FDR $<25 \%$ ) that would be expected of an overactivated PI3K/AKT pathway. Red indicates upregulated expression in $A$ and $A B$ thymomas and green indicates downregulated expression in $A$ and $A B$ thymomas. (B) Protein ELISA for activated phosphoAKT (Ser 473), and PTEN in a validation set of 35 thymomas and 3 normal tissues.

In our cell-based in vitro studies we demonstrate a significant inhibition of cell viability using these agents with our best $\mathrm{IC}_{50}$ approaching $100 \mathrm{~nm}$. Although the majority of type $\mathrm{A}$ and $\mathrm{AB}$ thymomas have a relatively benign course (Okuma et al, 2014), treated effectively with surgery, a portion can metastasise, primarily to the pleura (Vladislav et al, 2012). Our data suggest that a clinical trial of PI3K inhibitors is well warranted for this patient population with a phase II trial recently initiated in relapsed or refractory thymomas (http://clinicaltrials.gov/ct2/ show/NCT02220855).

Finally, significant controversy exists around the histological subtyping of thymomas with significant discordance among pathologists (Suster and Moran, 2006; Zucali et al, 2013). Our data demonstrate that the underlying gene expression of these tumours is $100 \%$ concordant with histological subtyping by an experienced pathologist who specialises in thymoma (SS Badve). Such expertise is not readily available at many institutions and thus a molecular classification may be useful to increase homogeneity across institutions. This is important for a rare disease, where much of the prognostic data are derived in cumulative from various cancer centres. Because C19MC expression is largely restricted to type $\mathrm{A}$ and $\mathrm{AB}$ thymomas, measurement of this microRNA cluster as a routine molecular diagnostic in thymoma tissues may be well warranted and potentially superior to histological subtyping by microscopy. As future studies to characterise potential subtype-specific targets, like PI3K, are elucidated, accurate clinical diagnostics of WHO subtype will be essential for a precision medicine approach in this disease. In conclusion, we demonstrate that overexpression of the largest miRNA cluster in the human genome on chromosome $19 \mathrm{q} 13.42$ is a transcriptional hallmark of type $\mathrm{A}$ and $\mathrm{AB}$ thymomas, and treatment of these tumours with PI3K pathway inhibitors may be an effective novel therapy. Outcomes from our clinical trial, as well as studies of the C19MC cluster with PI3K pathway inhibitors in other cancer types, will help to shed light on the utility of C19MC as a therapeutic biomarker. 


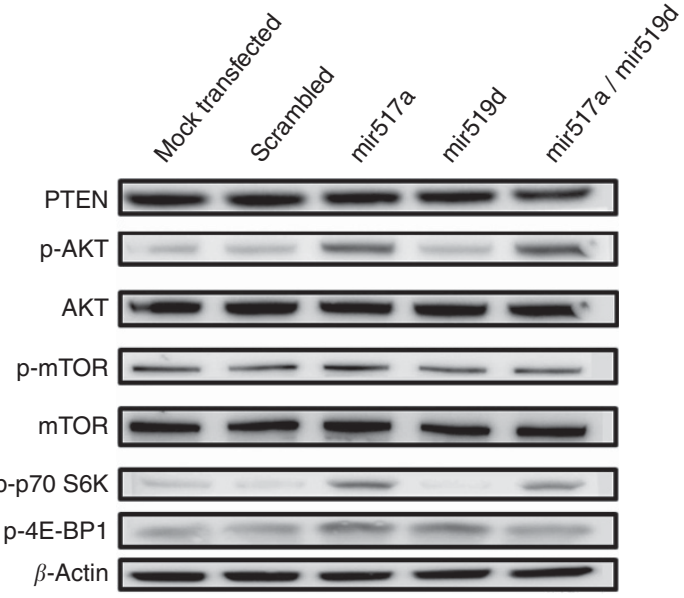

Figure 4. Functional analysis of key miRNAs within the C19MC cluster. The IU-TAB1 cell line was transfected with mir-517a, mir-519d, or the combination, along with a scrambled miRNA and mock transfected negative controls. The PTEN, phospho (p)-AKT (Ser 473), AKT (panAKT1/2/3), phospho-mTOR (Ser 2448), mTOR, phospho-p70 S6K (Ser 371), phospho-4E-BP1 (Thr 37/46), and $\beta$-actin protein levels were analysed using western blot.

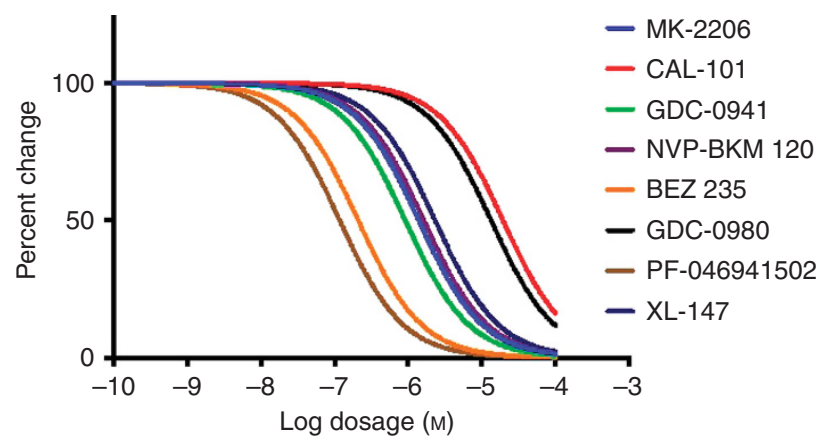

Figure 5. The thymoma AB cell line, IU-TAB 1, was tested with increasing log-doses of various PI3K and dual PI3K/mTOR inhibitors currently in phase 2 or 3 clinical trial. Cells were treated for $72 \mathrm{~h}$. The $y$ axis represents percent change in cell viability. The dual PI3K/mTOR inhibitors, PF-04691502 and BEZ235, demonstrated the best reduction in cell viability at an $\mathrm{IC}_{50}$ of 118 and $210 \mathrm{~nm}$, respectively.

\section{ACKNOWLEDGEMENTS}

We personally thank Dr Yesim Gokmen-Polar for providing the IU-TAB1 cell line. We also thank the Hochberg Family Foundation for establishing the Thymoma Tissue Repository at the IUSCC and the Wm P Loehrer Family Fund. We also thank the IUSCC Tissue Procurement and Distribution Core for providing the tissues for this study. Funding for this study was provided by NIH/NCI Public Health Service Grant No. P30 CA082709-12 (to PJ Loehrer).

\section{CONFLICT OF INTEREST}

M Radovich has received honoraria from Life Technologies Corporation. J Zhu is a former employee of Cofactor Genomics, LLC. J Glasscock is currently an employee, owner, and CEO of Cofactor Genomics, LLC.

\section{REFERENCES}

Brunet A, Bonni A, Zigmond MJ, Lin MZ, Juo P, Hu LS, Anderson MJ, Arden KC, Blenis J, Greenberg ME (1999) Akt promotes cell survival by phosphorylating and inhibiting a Forkhead transcription factor. Cell 96(6): 857-868.

Bullerdiek J, Flor I (2012) Exosome-delivered microRNAs of "chromosome 19 microRNA cluster" as immunomodulators in pregnancy and tumorigenesis. Mol Cytogenet 5(1): 27.

Dadmanesh F, Sekihara T, Rosai J (2001) Histologic typing of thymoma according to the new World Health Organization classification. Chest Surg Clin N Am 11(2): 407-420.

Engels EA (2010) Epidemiology of thymoma and associated malignancies. $J$ Thorac Oncol 5(10 Suppl 4): S260-S265.

Fine B, Hodakoski C, Koujak S, Su T, Saal LH, Maurer M, Hopkins B, Keniry M, Sulis ML, Mense S, Hibshoosh H, Parsons R (2009) Activation of the PI3K pathway in cancer through inhibition of PTEN by exchange factor P-REX2a. Science 325(5945): 1261-1265.

Flor I, Bullerdiek J (2012) The dark side of a success story: microRNAs of the C19MC cluster in human tumours. J Pathol 227(3): 270-274.

Fornari F, Milazzo M, Chieco P, Negrini M, Marasco E, Capranico G, Mantovani V, Marinello J, Sabbioni S, Callegari E, Cescon M, Ravaioli M, Croce CM, Bolondi L, Gramantieri L (2012) In hepatocellular carcinoma miR-519d is up-regulated by p53 and DNA hypomethylation and targets CDKN1A/p21, PTEN, AKT3 and TIMP2. J Pathol 227(3): 275-285.

Girard N, Shen R, Guo T, Zakowski MF, Heguy A, Riely GJ, Huang J, Lau C, Lash AE, Ladanyi M, Viale A, Antonescu CR, Travis WD, Rusch VW, Kris MG, Pao W (2009) Comprehensive genomic analysis reveals clinically relevant molecular distinctions between thymic carcinomas and thymomas. Clin Cancer Res 15(22): 6790-6799.

Gokmen-Polar Y, Sanders KL, Goswami CP, Cano OD, Zaheer NA, Jain RK, Kesler KA, Nelson Jr RP, Vance GH, Smith D, Li L, Cardoso AA, Badve S, Loehrer Sr PJ, Sledge GW Jr (2012) Establishment and characterization of a novel cell line derived from human thymoma $\mathrm{AB}$ tumor. Lab Invest 92(11): 1564-1573.

Hamaji M, Ali SO, Burt BM (2014) A meta-analysis of surgical versus non;surgical management of recurrent thymoma. Ann Thorac Surg 98(2): 748-755.

Jain RK, Mehta RJ, Henley JD, Kesler KA, Loehrer PJ, Badve S (2010) WHO types A and AB thymomas: not always benign. Mod Pathol 23(12): 1641-1649.

Kapur RP, Berry JE, Tsuchiya KD, Opheim KE (2014) Activation of the chromosome 19q microRNA cluster in sporadic and androgeneticbiparental mosaicism-associated hepatic mesenchymal hamartoma. Pediatr Dev Pathol 17(2): 75-84.

Kleinman CL, Gerges N, Papillon-Cavanagh S, Sin-Chan P, Pramatarova A, Quang DA, Adoue V, Busche S, Caron M, Djambazian H, Bemmo A, Fontebasso AM, Spence T, Schwartzentruber J, Albrecht S, Hauser P, Garami M, Klekner A, Bognar L, Montes JL, Staffa A, Montpetit A, Berube P, Zakrzewska M, Zakrzewski K, Liberski PP, Dong Z, Siegel PM, Duchaine T, Perotti C, Fleming A, Faury D, Remke M, Gallo M, Dirks P, Taylor MD, Sladek R, Pastinen T, Chan JA, Huang A, Majewski J, Jabado N (2014) Fusion of TTYH1 with the C19MC microRNA cluster drives expression of a brain-specific DNMT3B isoform in the embryonal brain tumor ETMR. Nat Genet 46(1): 39-44.

Korshunov A, Remke M, Gessi M, Ryzhova M, Hielscher T, Witt H, Tobias V, Buccoliero AM, Sardi I, Gardiman MP, Bonnin J, Scheithauer B,

Kulozik AE, Witt O, Mork S, von Deimling A, Wiestler OD, Giangaspero F, Rosenblum M, Pietsch T, Lichter P, Pfister SM (2010) Focal genomic amplification at 19q13.42 comprises a powerful diagnostic marker for embryonal tumors with ependymoblastic rosettes. Acta Neuropathol 120(2): 253-260.

Kozomara A, Griffiths-Jones S (2014) miRBase: annotating high confidence microRNAs using deep sequencing data. Nucleic Acids Res 4(Database issue): D68-D73.

Moran CA, Suster S (2008) The World Health Organization (WHO) histologic classification of thymomas: a reanalysis. Curr Treat Options Oncol 9(4-6): 288-299.

Okuma Y, Hosomi Y, Watanabe K, Yamada Y, Horio H, Maeda Y, Okamura T, Hishima T (2014) Clinicopathological analysis of thymic malignancies with a consistent retrospective database in a single 
institution: from Tokyo Metropolitan Cancer Center. BMC Cancer 14: 349.

Rippe V, Dittberner L, Lorenz VN, Drieschner N, Nimzyk R, Sendt W, Junker K, Belge G, Bullerdiek J (2010) The two stem cell microRNA gene clusters C19MC and miR-371-3 are activated by specific chromosomal rearrangements in a subgroup of thyroid adenomas. PLoS One 5(3): e9485.

Rodon J, Dienstmann R, Serra V, Tabernero J (2013) Development of PI3K inhibitors: lessons learned from early clinical trials. Nat Rev Clin Oncol 10(3): 143-153.

Shelly S, Agmon-Levin N, Altman A, Shoenfeld Y (2011) Thymoma and autoimmunity. Cell Mol Immunol 8(3): 199-202.

Suster S, Moran CA (2006) Thymoma classification: current status and future trends. Am J Clin Pathol 125(4): 542-554.

Travis WD, Brambilla E, Muller-Hermelink HK, Harris CC (eds) (2004) Pathology and genetics of tumours of the lung, pleura, thymus and heart. In: World Health Organization Classification of Tumours. IARC Press: Lyon, France.

Vaira V, Elli F, Forno I, Guarnieri V, Verdelli C, Ferrero S, Scillitani A, Vicentini L, Cetani F, Mantovani G, Spada A, Bosari S, Corbetta S (2012) The microRNA cluster C19MC is deregulated in parathyroid tumours. J Mol Endocrinol 49(2): 115-124.
Vladislav T, Jain RK, Alvarez R, Mehta RJ, Gokmen-Polar Y, Kesler KA, Henley JD, Loehrer Sr PJ, Badve S (2012) Extrathoracic metastases of thymic origin: a review of 35 cases. Mod Pathol 25(3): 370-377.

Wick MR (2008) Prognostic factors for thymic epithelial neoplasms, with emphasis on tumor staging. Hematol Oncol Clin North Am 22(3): 527-542.

Wick MR (2010) Histopathologic prognosis of thymomas: another example of medical surrogacy. Am J Clin Pathol 134(5): 703-705.

Zhang R, Wang YQ, Su B (2008) Molecular evolution of a primate-specific microRNA family. Mol Biol Evol 25(7): 1493-1502.

Zucali PA, Di Tommaso L, Petrini I, Battista S, Lee HS, Merino M, Lorenzi E, Voulaz E, De Vincenzo F, Simonelli M, Roncalli M, Giordano L, Alloisio M, Santoro A, Giaccone G (2013) Reproducibility of the WHO classification of thymomas: practical implications. Lung Cancer 79(3): 236-241.

This work is published under the standard license to publish agreement. After 12 months the work will become freely available and the license terms will switch to a Creative Commons AttributionNonCommercial-Share Alike 4.0 Unported License.

Supplementary Information accompanies this paper on British Journal of Cancer website (http://www.nature.com/bjc) 